David C. Chung MD FFarCs FRCPC, Dennis J. Hunter RRT, Franco J. Pavan RRT

\title{
The quick-mount pipeline connector: failure of a "fail-safe" device
}

This report describes the absence of gas flow despite the apparent proper mounting of a Canadian Liquid Air nitrous oxide quick-mount connector to its wall outlet. A similar but potentially more dangerous malfunction can occur with oxygen quick-mount connectors. The nature and cause of this defect are examined.

We observed a case in which there was total absence of gas flow despite apparent proper mounting of the nitrous oxide quick-mount connector to its wall outlet. This incident is reported here and to the Health Protection Branch through the Medical Devices Adverse Experience Reporting Project (MDAERP) of the Canadian Medical Association.

\section{Case report}

The device in question is a nitrous oxide quickmount connector (Canadian Liquid Air) illustrated in Figure 1. Coupling of this connector is achieved by plunging the male component into its female counterpart and turning it in a clockwise direction. The plunging manoeuvre will disengage the checkvalve to allow free passage of nitrous oxide, and the clockwise turn will secure the two parts together by sliding the retaining pin into the retaining groove. Malfunction of the nitrous oxide quick-mount connector was suspected during a routine inspection

\section{Key words}

EQUIPMENT: gas pipeline connectors, quick-mount connectors.

From the Department of Anaesthesia, University of Western Ontario, University Hospital, P.O. Box 5339, Station "A", London, Ontario N6A 5A5, where correspondence should be addressed to Dr. Chung. of the anaesthetic machine in preparation for an operation because there was no nitrous oxide flowing from the pipeline to the flowmeter. Since the cause of the malfunction was not immediately apparent the use of the operating room was abandoned, pending further investigation.

The nitrous oxide hose and its connectors to the wall outlet and the anaesthetic machine were examined in detail. The gas hose was intact; the diameter index safety system connecting the hose to the anaesthetic machine had not been tampered with; the wall connector seemed properly mounted by visual inspection and could be disconnected and reconnected with ease. Only after repeated trials did it become apparent that the male component of this nitrous oxide quick-mount connector could be plunged into its female counterpart and locked into place with a clockwise turn at two different depths. When the male component was pushed deep into its female counterpart, there was absolute resistance to further advancement together with an audible leakage of gas before the retaining pin could be locked into place. At a more superficial level of engage-

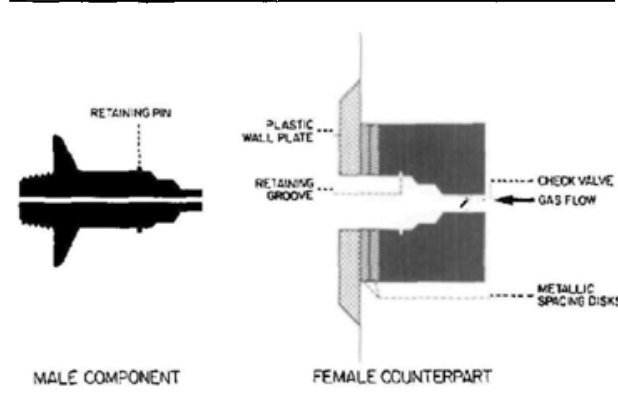

FIGURE 1 A diagram illustrating the male component and the female counterpart of the quick-mount pipeline connector in question. 


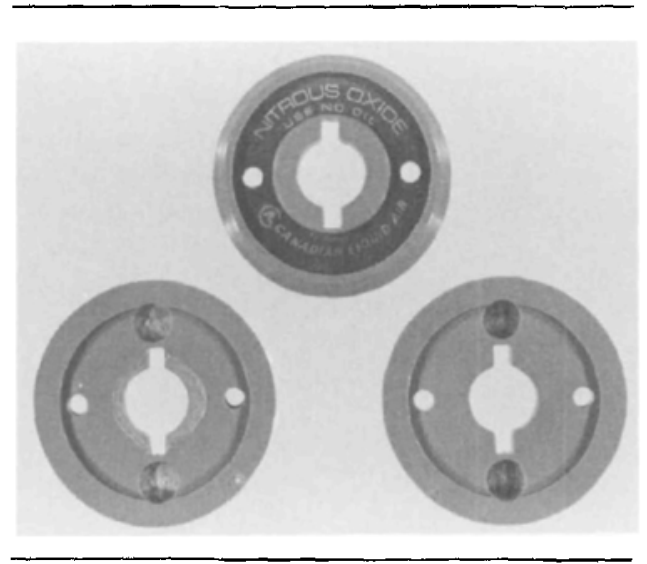

FIGURE 2 The face of the plastic wall plate (top), reverse of the plastic wall plate with the gouged-out defect (bottom left), and reverse of a wall plate without the defect (bottom right).

ment, resistance to further advancement of the male component was not absolute and there was no audible leakage of gas. Coupling at this more superficial level could be achieved only be executing the plunging and clockwise rotation motions simultaneously. Nitrous oxide would flow only after coupling was established at the deeper level.

On dismantling the gas outlet, the cause of malfunction became immediately apparent. There was a defect on the back of the plastic wall plate around its opening (Figure 2) and another on the front of the first metallic spacing disk (Figure 3), thus creating a false retaining groove between the

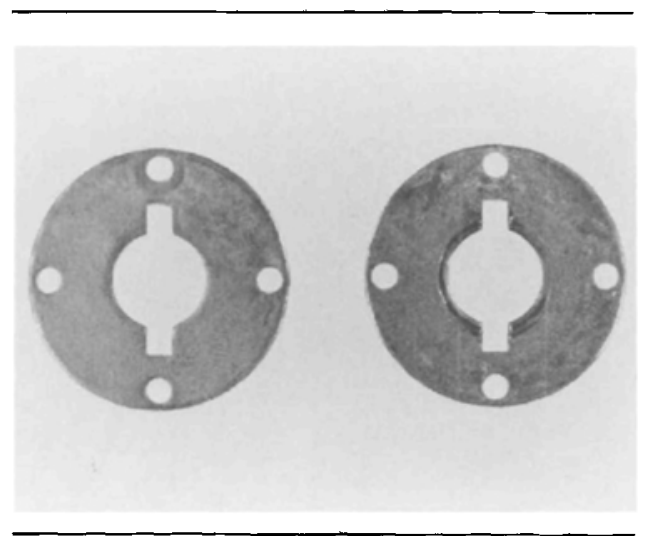

FIGURE 3 Metallic spacing disk with the gouged-out defect (right) and one without defect (left). wall plate and the first metallic spacing disk. Although apparent connection could be achieved with the retaining pin engaging this false retaining groove, the male component was not long enough to open the check-valve. This malfunction was confirmed by the absence of gas flow but could not be detected by the external appearance of the mounted connector alone. The defects on the plastic wall plate and on the spacing disk could only have been gouged out by the retaining pin - created by plunging the male component into its female counterpart and executing a clockwise twist simultaneously before the check-valve was opened.

With this failure in mind, wall outlets in all 16 operating and induction rooms in our institution were examined and a similar defect in another nitrous oxide outlet was discovered.

\section{Discussion}

In order to protect our patients from anaesthetic misadventure, increased attention has been paid to proper design of anaesthetic equipment and appropriate use of monitoring devices. Many of these devices are said to be "fail-safe"; but the use of this adjective is misleading: although they may serve to prevent a specific mishap, they can fail in other ways due to weaknesses in design or in the choice of construction material. The use of brittle plastic material as the wall plate and the necessity for spacing disks are weaknesses in the design of the nitrous oxide quick-mount connector described.

Like other anaesthetic mishaps, however, the importance of human error as a contributing cause to equipment failure should not be discounted., ${ }^{1,2}$ Proper coupling of this connector should be achieved only by pushing the male component straight into its female counterpart until the checkvalve is disengaged (as indicated by a gas leak) before turning this male component clockwise so as to slide the retaining pin into the retaining groove, Earlier rotation of the male component can cause the retaining pin to lodge in the potential space between the wall plate and the first metallic spacing disk or between the spacing disks themselves. Normally the retaining pin is lodged securely in the retaining groove after a 45 degree clockwise turn. If the male component can be rotated more than 45 degrees after engaging its female counterpart, the probability that the retaining pin has entered a false passage should be considered. We wish to 
emphasize that the reported incident occurred during an intercurrent period between regular inspections and services as recommended by the Canadian Standards Association (Nonflammable Medical Gas Piping System: Z305.1-M1984). In any case periodic inspections cannot replace proper procedures for testing the anaesthetic machine and its gas supplies before each case. Although we have described this malfunction of the quick-mount connector for nitrous oxide, a potentially more serious failure could occur to the oxygen connector. No equipment is fail-safe or fool-proof.

\section{References}

1 Cooper JB, Newbower RS, Long CD, MCPeek BJ. Preventable anesthesia mishaps: a study of human factors. Anesthesiology 1978; 49: 339-406.

2 Cooper JB, Newbower RS, Kitz RJ. An analysis of major errors and equipment failures in anesthesia management: considerations for prevention and detection. Anesthesiology 1984; 60: 34-42.

\section{Résumé}

Ce rapport fait part de l'absence de flot gazeux de protoxyde d' azote malgré un montage apparemment adéquat du connecteur "quick-moun" de la Compagnie Air Liquide du Canada à sa prise murale. Un malfonctionnement similaire mais potentiellement plus dangereux pourrait survenir avec les connecteurs "quick-mount" d'oxygène. La nature et la cause de cet accident som examinées. 